\title{
Gartanin induces autophagy through JNK activation which extenuates caspase-dependent apoptosis
}

\author{
MUN-OCK KIM ${ }^{1}$, HYUN-SUN LEE ${ }^{1}$, YOUNG-WON CHIN ${ }^{2}$, DONG-OH MOON ${ }^{3}$ and JONG-SEOG AHN ${ }^{1}$ \\ ${ }^{1}$ Korea Research Institute of Bioscience and Biotechnology (KRIBB), Ochang, ChungBuk 363-883; \\ ${ }^{2}$ Dongguk University-Seoul, Gyeonggi-do, Seoul 410-820; ${ }^{3}$ Department of Biology Education, \\ Daegu University, Gyungsan, Gyeongbuk 712-714, Republic of Korea
}

Received September 30, 2014; Accepted April 8, 2015

DOI: $10.3892 /$ or.2015.3948

\begin{abstract}
Hepatocellular carcinoma (HCC) is the third leading cause of cancer-related death worldwide. Development of novel agents to eradicate liver cancer cells is required for treatment of HCC. Gartanin, a xanthone-type compound isolated from mangosteen, is known to possess potent antioxidant, anti-inflammatory, antifungal and antineoplastic properties. In the present study, we investigated the cytotoxic effect of gartanin on HCC and explored the cell death mechanism. We showed that gartanin induced both the extrinsic and intrinsic apoptotic pathways, which were interconnected by caspase-8, - 9 and -3 activation. We also provided convincing evidence that gartanin induced autophagy in various cancer cells, as demonstrated by acridine orange staining of intracellular acidic vesicles, the degradation of p62, the conversion of LC3-I to LC3-II and GFP-LC3 punctate fluorescence. Additionally, gartanin induced the formation of typical autophagosomes and autolysosomes and enhanced the degradation rate of intracellular granule(s), including mitochondria. Notably, gartanin-mediated apoptotic cell death was further potentiated by pretreatment with autophagy inhibitors (3-methyladenine and bafilomycin A1) or small interfering RNAs against the autophagic genes (Atg5). These findings suggested that gartanin-mediated autophagic response protected against eventual cell death induced by gartanin. Moreover, gartanin treatment led to phosphorylation/activation of JNK and JNK-dependent phosphorylation of Bcl-2. Importantly, JNK inhibitor (SP600125) inhibited autophagy
\end{abstract}

Correspondence to: Professor Dong-Oh Moon, Department of Biology Education, Daegu University, Gyungsan, Gyeongbuk 712-714, Republic of Korea

E-mail: domoon@daegu.ac.kr

Dr Jong-Seog Ahn, Korea Research Institute of Bioscience and Biotechnology (KRIBB), Ochang, ChungBuk 363-883, Republic of Korea

E-mail: jsahn@kribb.re.kr

Key words: gartanin, apoptosis, autophagy, JNK, hepatocellular carcinoma yet promoted gartanin-induced apoptosis, indicating a key requirement of the JNK-Bcl-2 pathway in the activation of autophagy by gartanin. Taken together, our data suggested that the JNK-Bcl-2 pathway was the critical regulator of gartanininduced protective autophagy and a potential drug target for chemotherapeutic combination.

\section{Introduction}

Programmed cell death (PCD) is important in various developmental pathways. Apoptosis, the best known type, is now designated as type I PCD that eliminates damaged and infected cells, as well as regulates tissue homeostasis. Two major apoptotic pathways exist: the extrinsic pathway controlled by death receptors and the intrinsic pathway mediated by mitochondria. These apoptotic signaling pathways mediate an important cellular event, the activation of cysteine proteases and caspases that cleave different substrates eventually leading in cell death. However, there is considerable evidence to suggest that PCD is not confined to apoptosis but other mechanisms may also be involved. One of these mechanisms is 'autophagic PCD', which is a process that involves autophagosomes and autolysosomes $(1,2)$. Autophagosomes are double-membrane cytoplasmic vesicles that are designed to engulf various cellular components, including cytoplasmic organelles $(3,4)$. Autophagosomes fuse to lysosomes to become autolysosomes, which segregate cellular constituents for digestion prolonging survival for a short time under starvation conditions.

The molecular basis of autophagy has been extensively studied. Mammalian target of rapamycin (mTOR), a serine/threonine protein kinase, is one of the signaling proteins that initiate autophagy. mTOR negatively regulates autophagy related gene 1 (Atg1) or its mammalian homologs, Unc-151-like kinase (ULK-1 and -2) in nutrient rich conditions, thus inhibiting autophagy (5). During autophagosome formation, Beclin-1, a mammalian homolog of the yeast Atg6 gene has been shown to be involved in the nucleation step of the autophagosome. When Bcl-2 is phosphorylated by JNK, Beclin-1 is released from Bcl-2 at the level of the endoplasmic reticulum. Beclin-1 subsequently combines with the class III phosphatidylinositol 3-kinase Vps34, UVRAG and other partners required for autophagy vesicle nucleation $(6,7)$. The next step in autophagophore elongation requires conjugation of 
Atg5-Atg12 to localize at the outer membrane of the expending membrane. Atg8 (also called LC3-I) is cleaved by Atg4, and is subsequently conjugated with phophatidylethanolamine (PE) which leads to the LC3-II isoform. LC3-II is recruited both at the inner and the outer membranes of the growing vesicle. The completion of the autophagosome is followed by its fusion with a lysosome.

While hepatocellular carcinoma (HCC) is the most common malignancy worldwide, its incidence has been increasing in east Asia and Western countries $(8,9)$. Thus far the therapies of liver cancer have included surgery, chemical and target therapies, yet there is no perfect treatment (10). Therefore, it is necessary to identify novel therapeutic strategies for the management of HCC. Gartanin [1,3,5,8-tetrahydroxy2,4-bis(3-methyl-2-butenyl)] is a xanthone-type compound isolated from mangosteen, a tropical fruit native to southeast Asia. Gartanin has been reported to possess antioxidants, anticancer and antiproliferative properties $(11,12)$. Indeed, a recent study showed that gartanin sensitizes tumor necrosis factor (TNF)-related apoptosis-inducing ligand (TRAIL)-induced cell death through upregulation of DR5 expression in TRAIL-resistant human gastric adenocarcinoma cells (13). However, the anticarcinoma effect of gartanin on HCC and the molecular mechanism of its effect have not yet been fully determined.

The purpose of the present study was to determine whether gartanin induced PCD in HCC and to examine the relationship between autophagy and apoptosis. In the present study, we demonstrated for the first time that gartanin induced an autophagic response in various cancer cell lines. Furthermore, genetic inhibitors or inhibition of autophagy by specific chemical inhibitors aggravated cell death in response to gartanin stimulation. Furthermore, gartanin plus autophagy inhibitor (3-MA) caused marked HCC growth inhibition compared with single agents alone, suggesting that gartanin-induced autophagy may play a self-protective role against its own cytotoxic effect. Our data also showed that the autophagy induced by gartanin was independent of mTOR and was mediated through activation of JNK and subsequent phosphorylation of Bcl-2, ultimately leading to autophagydependent interference with gartanin-induced apoptosis. Thus, gartanin may has potential therapeutic use by targeting the autophagic pathway.

\section{Materials and methods}

Cell culture. Human hepatoma cell lines Hep3B, HepG2 and Huh7 were purchased from the American Type Culture Collection. Cells were cultured in Dulbecco's modified Eagle's medium (DMEM) supplemented with $10 \%$ fetal bovine serum (FBS) and antibiotics (Life Technologies) and maintained at $37^{\circ} \mathrm{C}$ with $5 \% \mathrm{CO}_{2}$ in a humidified atmosphere.

Chemicals. Gartanin isolated from mangosteen was kindly gifted by Professor Y.-W.C. (Dongguk University). Acridine orange (AO) and SP600125 were purchased from the Sigma Chemical Corporation. MitoTracker was purchased from Invitrogen. Anti-p62, c-Jun, Beclin-1 and LC3 antibodies were purchased from Santa Cruz Biotechnology. Anti-Atg5, phosphorylated JNK (T183/Y185), phospho-specific antibodies against c-Jun (S63), mTOR (S2448 and S2481) antibodies were purchased from Cell Signaling. Other antibodies were from Dakopatts.

Cell viability (MTT). Cell viability assays were conducted as previously described (14).

Observation of morphologic changes. Hep3B cells (5x105/well) were seeded into 6-well culture plates and incubated with gartanin for $24 \mathrm{~h}$. Cellular morphology was observed by a phase contrast microscope (Leica, Nussloch, Germany).

Transmission electron microscopy. Electron microscopy was as previously described (15). Briefly, cells were prefixed in Karnovsky's solution [1\% paraformaldehyde, $2 \%$ glutaraldehyde, $2 \mathrm{mmol} / 1$ calcium chloride, $0.1 \mathrm{~mol} / 1$ cacodylate buffer ( $\mathrm{pH}$ 7.4)] for $2 \mathrm{~h}$ and washed with cacodylate buffer. Postfixing was carried out in $1 \%$ osmium tetroxide and $1.5 \%$ potassium ferrocyanide for $1 \mathrm{~h}$. After dehydration with 50-100\% alcohol, the cells were embedded in Poly/Bed 812 resin (Pelco), polymerized and observed under an electron microscope (EM 902A; Zeiss).

Acidic vesicular organelles with AO staining. Gartanin-treated cells were stained with $1 \mu \mathrm{g} / \mathrm{ml} \mathrm{AO}$ for $15 \mathrm{~min}$ and cells were observed under a fluorescence microscope, to detect acidic vesicular organelles (AVO). Gartanin-treated cells were stained with AO, detached by trypsinization, and processed for the FACScan using CellQuest software (Becton-Dickinson) to quantify the development of AVOs.

Mitochondrial labelling within live cells. Gartanin-treated cells were stained with $1 \mu \mathrm{g} / \mathrm{ml}$ MitoTracker for $15 \mathrm{~min}$ and observed under a fluorescence microscope; samples were further analyzed by FACScan using CellQuest software.

GFP-LC3 translocation. Hep3B cells were transfected with the plasmid encoding GFP-LC3, and then treated with various dose of gartanin. Translocation of GFP-LC3 from cytosol to autophagic vacuoles was observed by fluorescence microscopy and the fluorescence intensity of GFP of LC3 was assessed by FACScan using CellQuest software.

Western blotting. Western blotting experiments were conducted as previously described in details (16).

Flow cytometric analysis. Cells were fixed with $1 \mathrm{U} / \mathrm{ml}$ of RNase A (DNase-free; Sigma) and $10 \mu \mathrm{g} / \mathrm{ml}$ of propidium iodide (PI) (Sigma-Aldrich) overnight at room temperature, in the dark. A flow cytometer was used to analyze the level of apoptotic cells containing sub-G1 DNA content. For Annexin V staining, live cells were incubated with Annexin V (R\&D Systems).

Statistical analysis. All data are presented as mean \pm SE of at least 3 independent experiments. The statistical significance of differences was assessed using ANOVA (GraphPad software) followed by the Student-Newman-Keuls' multiple comparison tests. $\mathrm{P}<0.05$ was considered to indicate a statistically significant result. 

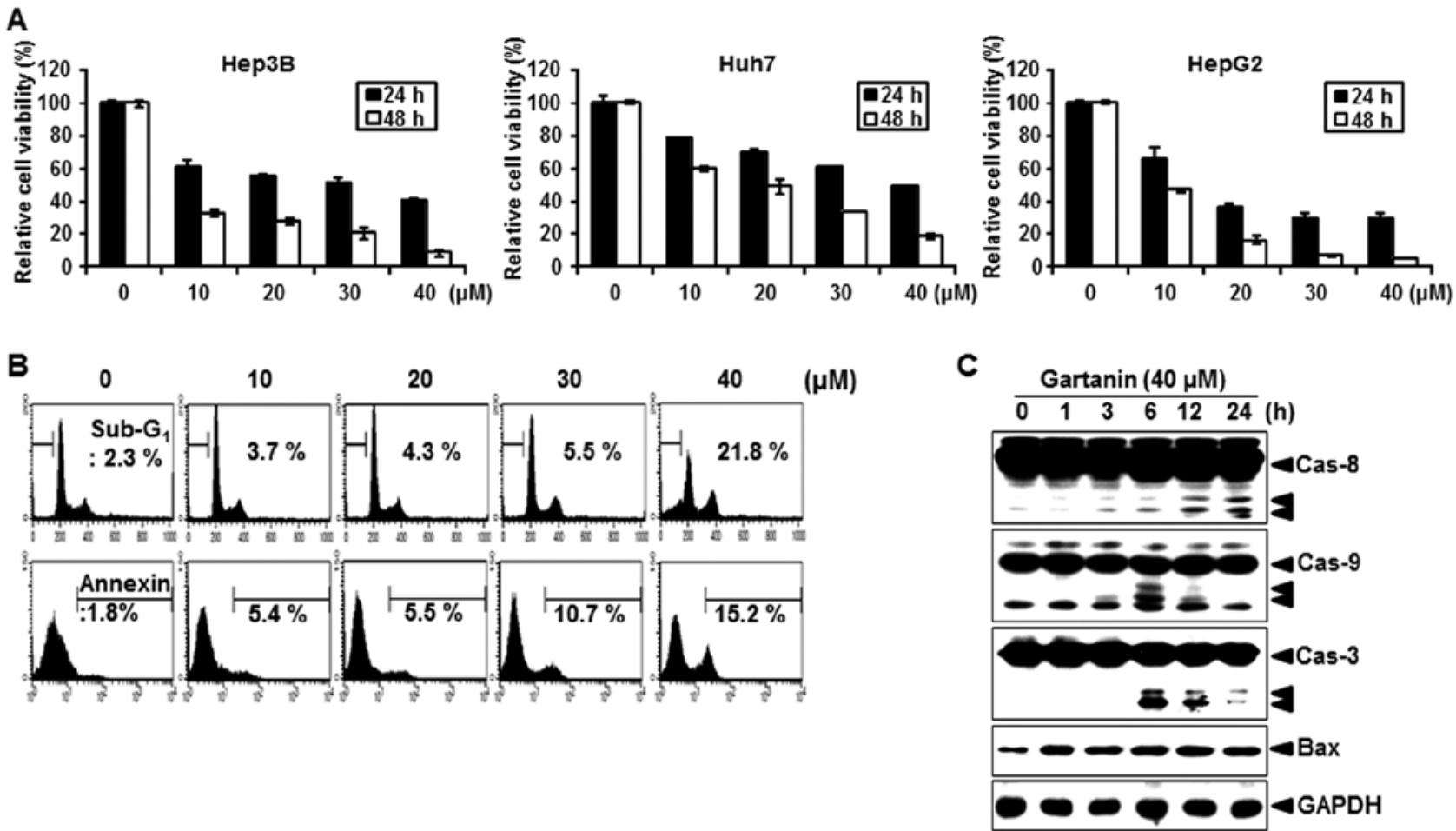

Figure 1. Gartanin induced cell death of HCC cells through caspase activation. (A) Human hepatocellular carcinoma Hep3B, HepG2 and Huh7 cells were treated with indicated concentrations of gartanin for $24 \mathrm{~h}$. MTT assay was performed to analyze cellular viability. (B) Flow cytometry analyses of the DNA content (upper) and Annexin $\mathrm{V}^{+}$(bottom) of the cells. (C) Western blot analyses of cell extracts (caspase- $8,-9$ and -3 , and Bax). GAPDH was used as a loading control.

\section{Results}

Gartanin inhibits proliferation of $\mathrm{HCC}$. We first evaluated the effect of gartanin on the proliferation of hepatoma cancer cells. Gartanin inhibited cell proliferation in a time- and dose-dependent manner in Hep3B, HepG2 and Huh7 cells (Fig. 1A). Our results revealed that $10-40 \mu \mathrm{mol} / 1$ gartanin decreased viability in the tested HCC cell lines, suggestive of the preferentially cytotoxicy of gartanin to malignant HCC cells. Next, we investigated whether decreased cell viability by gartanin treatment was due to the induction of apoptotic signaling. Treatment with gartanin for $24 \mathrm{~h}$ increased the accumulation of Hep3B cells in the sub-G1 phase (Fig. 1B, upper panel) and positive staining with Annexin V (Fig. 1B, lower panel). We also found that the activity of caspase-8, -9 and -3 was significantly increased on gartanin treatment (Fig. 1C). Furthermore, gartanin enhanced the expression of pro-apoptotic protein Bax (Fig. 1C). These results indicated that the classic mitochondrial apoptosis pathways were involved in gartanin-induced Hep3B cell apoptosis. Thus, gartanin induced apoptotic cell death in HCC suggestive of its potential as an effective therapeutic approach to malignant HCC.

Gartanin induces autophagy of HCC. We next determined whether gartanin could induce autophagy and apoptosis simultaneously. Gartanin-treated Hep3B cells showed numerous small vesicles around their nuclei on phase-contrast microscopy (Fig. 2A). We further investigated whether vacuolation was observed in gartanin-treated Hep3B cells. AO, a lysosomotropic agent, is able to stain the acidic vesicular organelle (AVOs) with cytoplasm fluorescence changing from bright green to bright red, thereby offering a rapid and quantitative measure of the induction of autophagy. FACS analysis showed that the percentage of cells with AVOs was dose-dependently higher in the gartanin-treated group vs. the control group (Fig. 2B). Vesicular accumulation of microtubule-associated protein 1 light chain 3 (LC3) is a marker of autophagy, therefore, in order to further evaluate autophagy, gartanin-treated cells were transfected with GFP-LC3 plasmid. GFP-LC3 was diffusely expressed in untreated cells, whereas gartanin-treated cells displayed a highly-intense punctate expression (Fig. 2C, upper panel). GFP-LC3 plasmid was also used in flow cytometry experiments to measure the turnover of autophagic vesicles. We found that gartanin significantly decreased the fluorescent intensity of GFP-LC3 via rapid degradation by the autolysosome (Fig. 2C, lower panel). These results suggested that fragmented intracellular granules may be engulfed by autophagic vacuoles, on treatment with gartanin. We furthermore assessed the effect of gartanin on the levels of Atg5, an autophagic marker (17), and the conversion of the cytoplasmic form of the LC3-I protein $(18 \mathrm{kDa})$ to the preautophagosomal and autophagosomal membrane-bound form of LC3-II (16 kDa). As shown in Fig. 3A, immunoblot analysis revealed that gartanin treatment dose- and time-dependently led to an increase in the levels of Atg5 and LC3-II proteins in Hep3B cells. Quantitative analysis also indicated that gartanin treatment led to an increase in Atg5 and LC3-II levels as early as $1 \mathrm{~h}$ after drug exposure and gradually increased thereafter. Next, we examined whether gartanin induced autophagy in other cell types. We found that gartanin increased LC3-II 
A

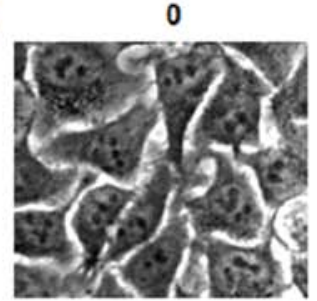

B

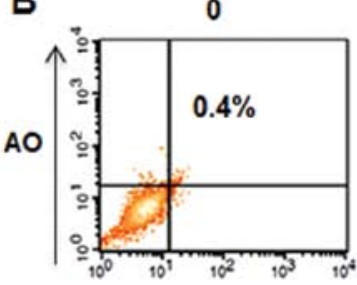

C
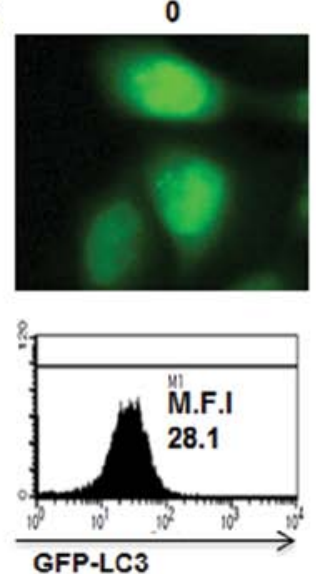

10

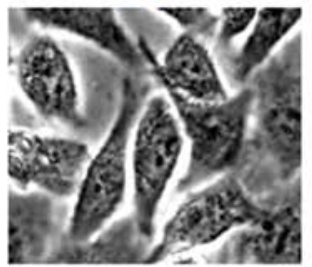

10

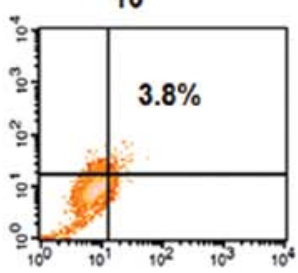

10
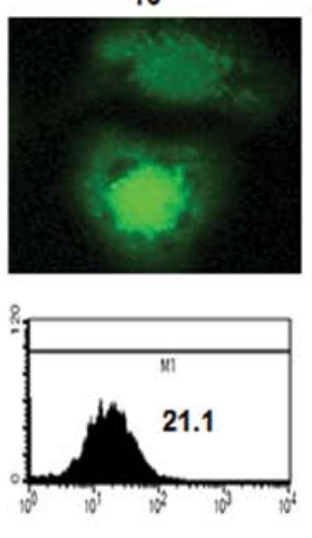

20

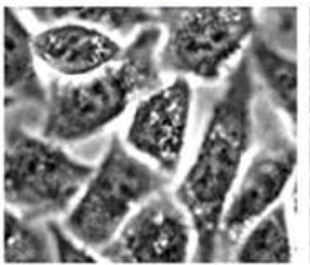

20

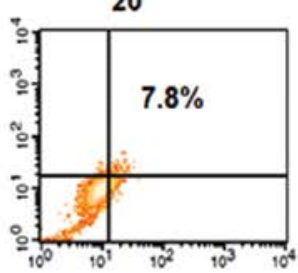

20
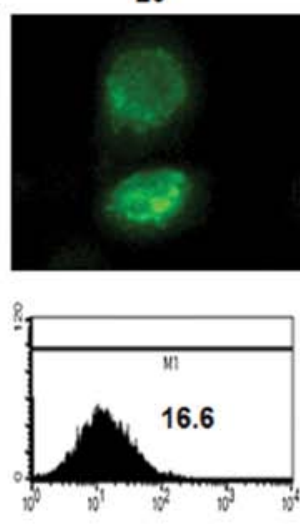

30

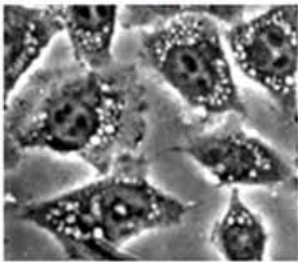

30

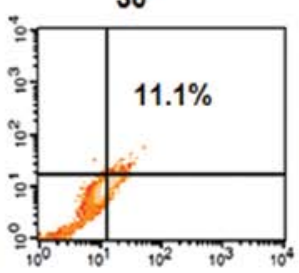

30
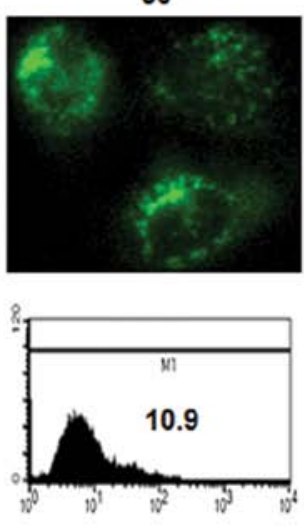

40 (Gartanin $\mu \mathrm{M})$

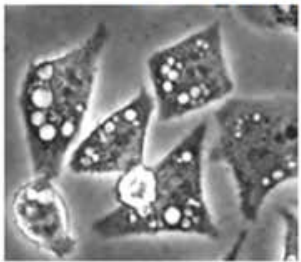

$40($ Gartanin $\mu M)$

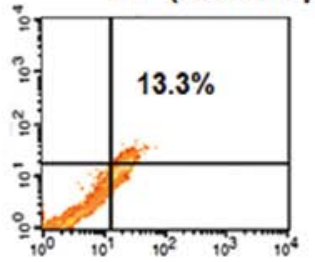

40 (Gartanin $\mu M)$
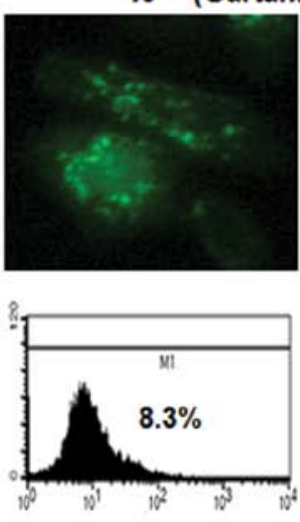

Figure 2. Induction of autophagy in HCC cells treated with gartanin. (A) Hep3B cells treated with up to $40 \mu \mathrm{mol} / 1$ gartanin for $24 \mathrm{~h}$ and observed under a phase-contrast microscope. (B) Flow cytometry analysis of fluorescent intensity of acridine orange (AO). (C) Hep3B cell lines stably transfected with GFP-LC3 were treated with indicated concentration of gartanin for $24 \mathrm{~h}$. Formation of GFP-LC3 vacuoles (dots) was detected after gartanin treatment for an additional $24 \mathrm{~h}$ under the fluorescent microscope (upper). The percentage of cells with GFP-LC3 fluorescence was calculated by randomly counting 10,000 cells by flow cytometry (lower).

A
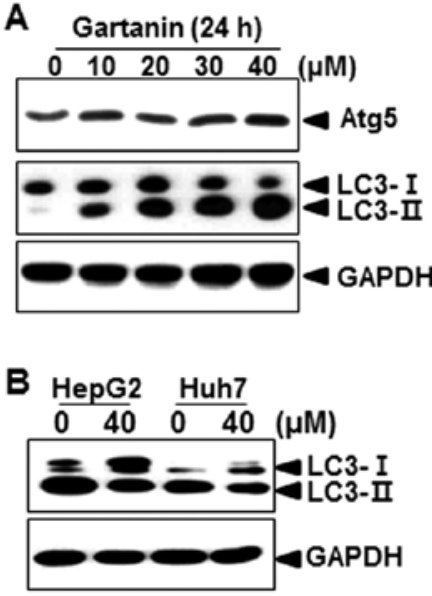
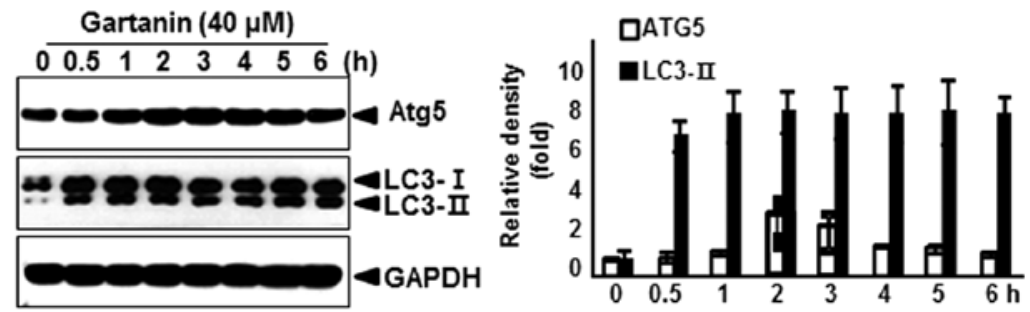

C WT MEF ATG5 $\%$ MEF

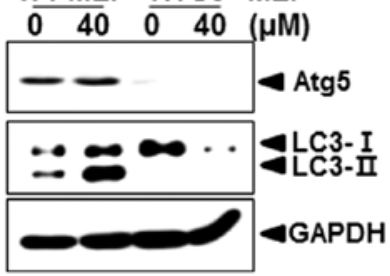

Figure 3. Gartanin induces activation of the autophagic signaling pathway. (A) Hep3B cells were treated with gartanin, and whole cell lysates were then subjected to immunoblotting with antibodies against Atg5, LC3 and GAPDH. Relative density indicated the relative ratio between LC3II isoforms and Atg5 normalized to GAPDH using laser densitometer. Data are presented as means \pm standard error (SE) and are representative of the results obtained from 3 independent experiments. (B) HepG2 and Huh7 cells were treated with gartanin and subsequently cell extracts were prepared for immunoblot analysis. (C) Wild-type MEF (WT MEF) or ATG5 knockout MEF (ATG5 ${ }^{-/}$MEF) cells were treated with $40 \mu \mathrm{mol} / 1$ gartanin for $24 \mathrm{~h}$. Activation of autophagy was determined by measuring the conversion of LC3I to LC3II by western blot analysis with the indicated antibodies. 
A
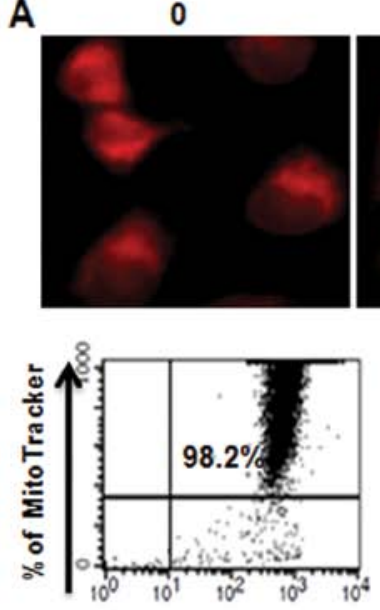

10
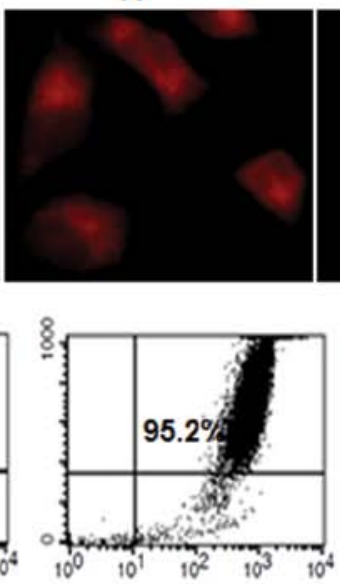

20
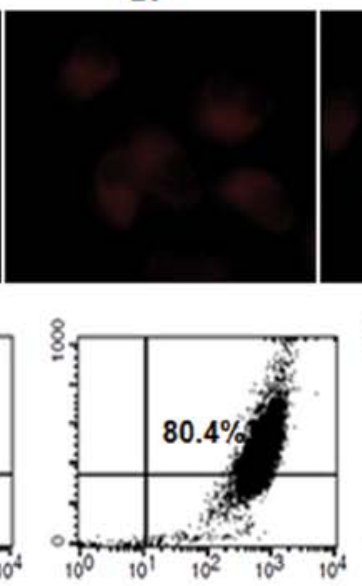

30
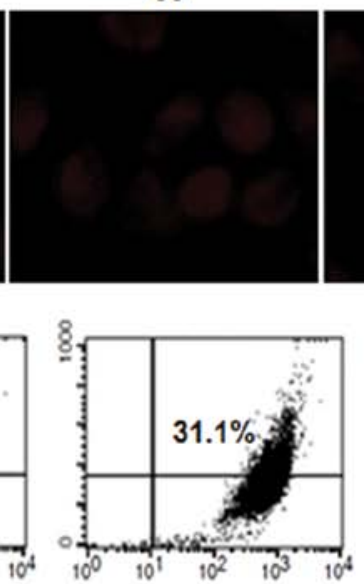

40 (Gartanin $\mu M)$
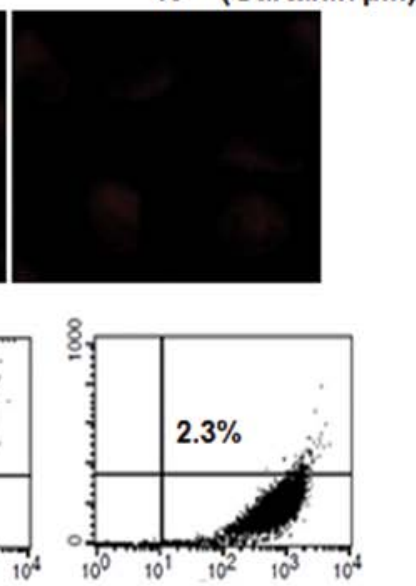

B
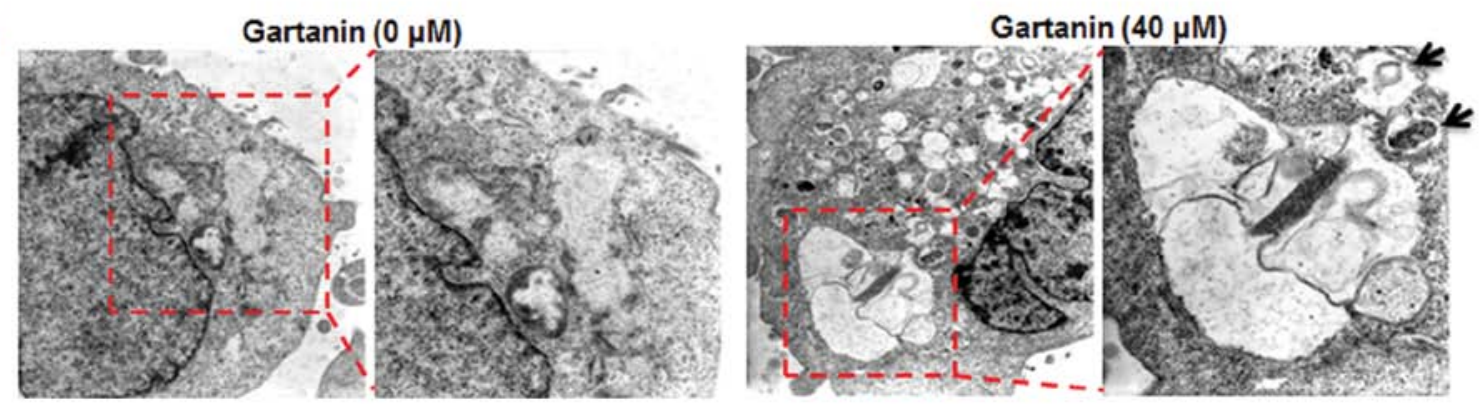

Figure 4. Gartanin-induced autophagy is preceded by functional and structural disruption of mitochondria. (A) Hep3B cells were treated with gartanin for $24 \mathrm{~h}$ and then incubated with MitoTracker. Fluorescence of MitoTracker was detected under the fluorescent microscope (upper). The percentage of cells with MitoTracker fluorescence was calculated by randomly counting 10,000 cells by flow cytometry (lower). (B) Hep3B cells were untreated or treated with $40 \mu \mathrm{mol} / 1$ gartanin for $24 \mathrm{~h}$ and transmission electron microscopy was carried out.

proteins in HepG2 and Huh7 cell lines (Fig. 3B). Wild-type mouse embryonic fibroblasts (WT MEF) and ATG5 knockout MEF (ATG5 ${ }^{-1} \mathrm{MEF}$ ) cells were incubated in the presence of gartanin, to determine the role of ATG5 in gartanin-induced autophagy. Autophagy was measured by immunoblot analysis of LC3. Gartanin failed to induce autophagy in ATG5 ${ }^{-} \mathrm{MEF}$ cells, while it dramatically induced autophagy in WT MEF cells (Fig. 3C). These results indicated that ATG5 played an important role in the induction of gartanin-mediated autophagy.

Gartanin-damaged mitochondria are targeted by autophagy. We next examined whether gartanin-induced autophagic cell death in Hep3B cells was associated with alterations in mitochondrial function and/or structure crucial to regulating cell death. Gartanin induced a dose-dependent significant loss of mitochondrial membrane potential (MMP) analyzed by MitoTracker on fluorescence microscopy (Fig. 4A, upper panel) and FACScan (Fig. 4A, lower panel). Electron microscopy subsequently provided further confirmation of gartanin-induced autophagy in Hep3B cells. Electron micrographs of untreated cells showed normal morphology of all organelles, with mitochondria scattered homogeneously throughout the cells. Gartanin treatment for $24 \mathrm{~h}$ resulted in more advanced mitochondrial disruption with frequent fusion between the vacuoles. Vacuoles appeared in increasing sizes signifying ongoing fusion of autophagosomes with lysosomes (Fig. 4B).
Autophagy inhibition enhances gartanin-induced apoptosis. Recent research suggests that inhibition of autophagy may enhance chemosensitization in human cancer cells. Accordingly, we investigated whether pharmacological inhibition of autophagy, enhanced gartanin-induced apoptosis. Firstly to inhibit autophagy, we utilized the class III phosphatidylinositol 3-kinase (PI3K) inhibitor 3-MA that was previously reported to sensitize cancer cells to chemotherapy-induced apoptosis. As shown in Fig. 5A, 3-MA plus gartanin treatment resulted in an increased percentage of apoptotic cancer cells than gartanin alone by a PI staining assay. Corroborating this finding, 3-MA enhanced caspase-3 cleavage, a marker of apoptosis (Fig. 5B). Baf A1, a specific inhibitor of vacuolar type $\mathrm{H}^{+}$-ATPase, prevents autophagy at the late stage by inhibiting fusion between autophagosomes and lysosomes. Baf A1 similarly enhanced gartanin-induced apoptotic signaling, as shown by caspase-3 cleavage (Fig. 5C and D). Consistent with the pharmacological approach, suppression of autophagy by silencing Atg5 aggravated gartanin-induced apoptosis in Hep3B cells as indicated by an increase in sub-G1 population and cleaved caspase-3 and PARP (data not shown). These results suggested that gartanin induced canonical autophagy, and induction of autophagy had a protective role in tumor cells subjected to the cytotoxicity of gartanin.

Gartanin-induced autophagy is mediated through the $J N K-B c l-2$ pathway. We analyzed the effect of gartanin on the main cellular signaling pathways that are constitutively modu- 

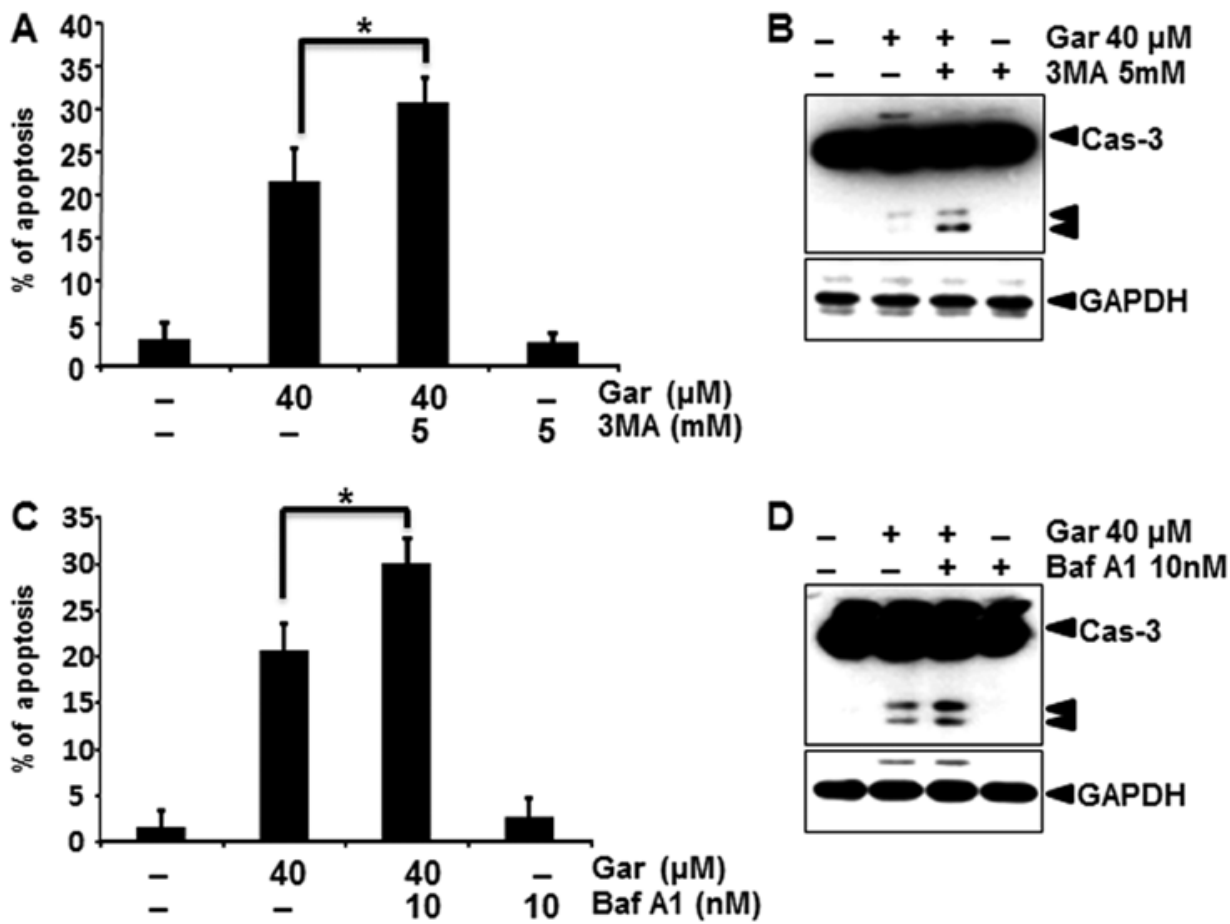

Figure 5. Inhibition of autophagy increased gartanin-induced cell death. (A-D) Hep3B cells were incubated with gartanin for $24 \mathrm{~h}$ in the absence or presence of autophagy inhibitors (5 mM 3-MA and $10 \mathrm{nM}$ Baf A1) pretreated for $1 \mathrm{~h}$. (A and C) Sub-G1 population after PI staining were designated as apoptotic cells Data are presented as means \pm standard error (SE) and are representative of the results obtained from 3 independent experiments. "p $<0.05$ vs. untreated control. (B and D) Immunoblotting was performed using antibodies for caspase-3, cleaved caspase-3 and GAPDH.

A Gartanin $(40 \mu \mathrm{M})$
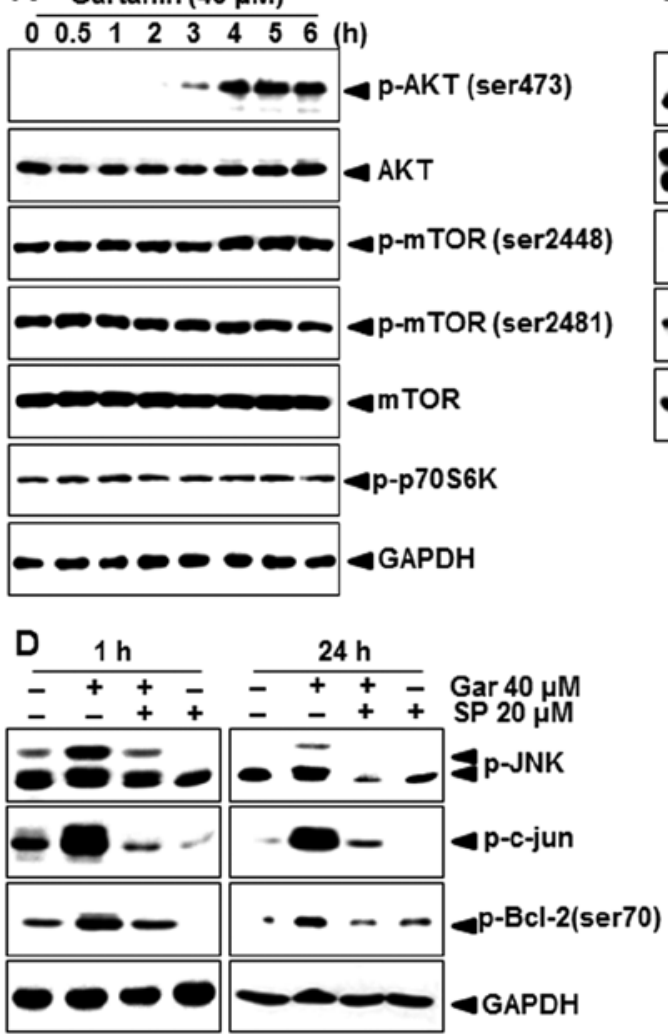

B Gartanin $(40 \mu \mathrm{M})$

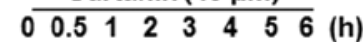

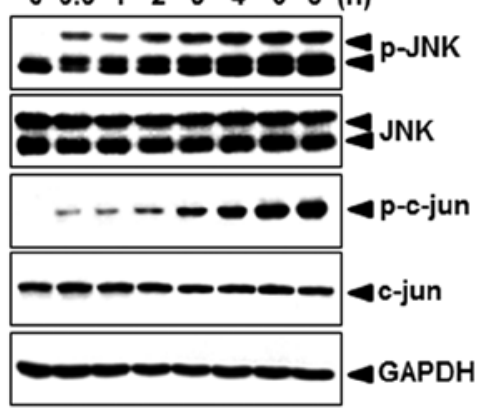

C $\operatorname{Gartanin}(\mathbf{4 0} \mu \mathrm{M})$

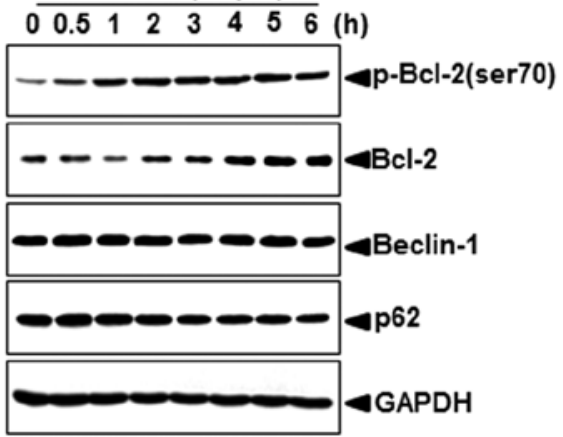

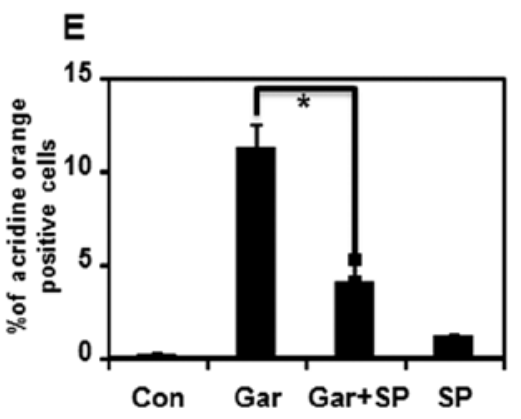

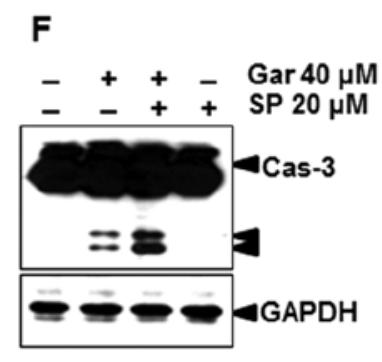

Figure 6. Autophagy-related signal transduction in Hep3B cells with gartanin treatment. (A-C) Hep3B cells were treated with indicated concentrations of gartanin for $6 \mathrm{~h}$, after which protein extracts were subjected to immunoblotting using the specific antibodies, including p-Akt (ser473), p-mTOR (ser2448 and ser2481), p-p70S6K, Beclin-1, p-JNK (Thr183/Tyr185), JNK, p-c-jun, p-Bcl-2 (ser70) and Bcl-2. (D) Hep3B cells were treated with $20 \mu \mathrm{mol} / 1$ SP600125 for $0.5 \mathrm{~h}$ prior to gartanin treatment for the indicated times. Total protein was subjected to 10\% SDS-PAGE followed by western blotting using anti-p-JNK, p-c-jun and p-Bcl-2. (E) SP600125 (20 $\mu \mathrm{mol} / \mathrm{l})$ pretreated Hep3B cells were treated $40 \mu \mathrm{mol} / 1$ gartanin for $24 \mathrm{~h}$. Following treatment, cells were stained with $1 \mathrm{mg} / \mathrm{ml}$ acridine orange (AO) at $37^{\circ} \mathrm{C}$ for $15 \mathrm{~min}$. Staining of AOs was analyzed using flow cytometry. (F) Cell extracts were used in western blot analyses. 
lated in Hep3B cells, to determine its mode of action. Since inhibition of the mTOR signaling pathway is a well-characterized mechanism in the initiation and maturation of autophagy, we examined whether gartanin regulated the mTOR signaling pathway. Unexpectedly, gartanin treatment up to $6 \mathrm{~h}$ induced a sharp increase in the phosphorylation of mTOR, as well as its downstream targets p70 ribosomal protein S6 kinase (p70S6K), indicating that gartanin induces autophagy through an Akt/mTOR-independent pathway (Fig. 6A). JNK has also been implicated in the induction of autophagy by various stimuli, including starvation (18), cytokine stimulation (19) and ER stress (20); hence, we next investigated whether JNK was involved in gartanin-induced autophagy. As shown in Fig. 6B, gartanin triggered JNK activation, within $0.5 \mathrm{~h}$ in Hep3B cells. This response was sustained for at least $6 \mathrm{~h}$ (Fig. 6B). Indeed, JNK activation correlated with an increase in c-Jun phosphorylation. One mechanism by which JNK contributes to autophagy is via phosphorylation of the anti-apoptotic protein Bcl-2 $(21,22)$ and thereby, its dissociation from Beclin-1 (23). Furthermore, activated JNK mediates transcriptional activity of Bcl-2 (24), p62 (25) and Beclin-1 resulting in autophagy (26). Western blotting was used to determine whether JNK activation stimulated phosphorylation and/or expression of these proteins. Gartanin unregulated the expression as well as phosphorylation of Bcl-2, but had no effect on p62 and Beclin-1 in our system (Fig. 6C). In order to further evaluate the relationship between gartanin-induced autophagy and the JNK signaling, cells were treated with gartanin in the presence of SP600125 (a JNK activity-specific inhibitor). Our data showed that pretreatment of SP600125 significantly inhibited the $24 \mathrm{~h}$ gartanin-induced phosphorylation of JNK, c-jun and Bcl-2 at 1 (Fig. 6D). Furthermore, gartanin-induced AVOs were decreased by SP600125 pretreatment (Fig. 6E). SP600125 mediated JNK inhibition stimulated gartanin-induced caspase-3 activation (Fig. 6F). Collectively, these results validated the requirement of JNK activation in stimulation of autophagy, and showed that JNK inhibition promoted gartanin-induced cell death.

\section{Discussion}

Cancer remains one of the most aggressive and lethal human malignancies worldwide. Although chemotherapy,radiotherapy and surgery have been used for many years, these anticancer therapies can only offer limited benefits to cancer patients due to metastasis, acquired chemoresistance and toxicity. Evidence from recent studies suggest that the consumption of vegetables and fruits decreases the incidence of cancer (27). Natural products may serve as novel adjunctive agents and chemoprevention of cancer. Mangosteen (Garcinia mangostana Linn.), a well-known tropical fruit contains considerable amounts of biologically active compounds, such as xanthones. Among the xanthones, gartanin is most abundant and frequently studied.

Autophagy is a degradative process in eukaryotic cells that results in the breakdown of intracellular material within lysosomes (28). It serves as an alternative route of PCD called type-2 PCD or autophagic cell death. Evidently autophagy is not always pro-death but instead can be pro-survival under conditions of cellular stress induced by nutrient deprivation or chemotherapy, thus allowing cells to evade apoptosis. In tumor cells, the role of autophagy may depend on tumor types, the stage of tumorigenesis, and the nature and extent of the insult (29).

In the present study, we have firstly demonstrated that gartanin induced apoptosis in Hep3B cells, through the classic apoptotic pathways. We also identified the process of autophagy in response to gartanin together with the underlying molecular mechanisms. In the present study, gartanin-induced autophagy was evidenced by autophagosomal marker LC3 conversion, accumulation of AO-labeled acidic vesicles consistent with autophagolysosomes, and punctate formation of GFP-LC3. We also showed that gartanin induced autophagy in human HCC cell lines, Hep3B, HepG2 and Huh7. These findings indicated that gartanin could generally induce autophagy in various cancer cells, and not in a strict cell type-specific manner. We used pharmacological and genetic approaches to inhibit autophagy, in our attempt to define a pro-survival or pro-cell death role for gartanin. 3-MA and Baf A1 were used as inhibitors of autophagy. Our results showed that 3-MA or Baf A1 treatment significantly enhanced gartanin-induced apoptosis in Hep3B cells, indicating that gartanin-mediated autophagy was protective. Furthermore, inhibition of autophagy-related genes (Atg5) resulted in significant apoptotic cell death in Hep3B cells treated with gartanin. Thus, we concluded that the gartanin-triggered autophagy had protective effects in various cancer cells and inhibition of autophagy could be a promising strategy to enhance the antitumor efficiency of gartanin.

We next investigated the potential pathways involved in gartanin-induced induction of autophagy, in an attempt to identify means to enhance the efficacy of gartanin. We found an important role for JNK signaling in gartanin-induced autophagy, in our system. JNK has been reported to have critical functions upstream of autophagy induced by growth factors, nutrients and stress, including hypoxia and oxidative stress. It is well established that the activation of JNK, also referred to as stressactivated kinases, mediates Bcl-2 phosphorylation (23). Bcl-2 phosphorylation disrupts the interactions between Beclin-1 and Bcl-2, releasing Beclin-1 to promote autophagy. Our data showed that gartanin enhanced the phosphorylation of JNK and Bcl-2 in Hep3B cells. Additionally, SP600125-mediated inhibition of JNK abrogated gartanin-induced autophagy via dephosphorylation of JNK and Bcl-2. Thus, it was reasonable to hypothesize that gartanin treatment may induce JNK activation, which results in Bcl-2 phosphorylation, thereby disrupting Bcl-2 -Beclin-1 interaction, with net stimulation of autophagy. JNK also mediates the late phase of autophagy through regulation of microtubule stability via phosphorylation of MAP1B (microtubule-associated protein1B) which mediates autophagosome trafficking to the lysosome through interaction with both LC3-I and LC3-II (30). Hence, further study is required to investigate the role of JNK in MAP1B activation. We also found that gartanin upregulated the gene expression of anti-apoptotic Bcl-2. JNK is known to induce activation of activator protein-1 (AP-1), hence an interesting possibility exists for AP-1 activation in gartanin-mediated Bcl-2 regulation. More prudent experiments will be needed for elucidating the role of AP-1 on gartanin-induced Bcl-2 expression. Summarily, we cautiously concluded that gartanin-induced JNK activation may have an anti-apoptotic action via upregulation and phosphorylation of $\mathrm{Bcl}-2$. 
Additionally, the Akt/mTOR pathway is the classic pathway often involved in regulating autophagy. Inhibition of the Akt/mTOR pathway has consistently been associated with triggering autophagy in cancer cells. Rapamycin is the mTORC1 inhibitor and induces autophagy in different tumor cells. However, in the present study, we found that the expression of phospho-mTOR (ser2448), as well as the levels of phosphorylated p70S6 kinase was slightly increased in Hep3B cells after exposure to gartanin. These results suggested that gartanin induced autophagy via an mTOR-independent pathway.

In conclusion, we demonstrated that autophagy triggered by gartanin occurred in many types of cancer cells. In addition, our data showed that activation of JNK signaling pathway was responsible for gartanin-induced autophagy via increasing phosphorylation of $\mathrm{Bcl}-2$ which promoted its release from Beclin-1. Thus, our data suggested that the JNK-Bcl-2 pathway was the critical regulator of gartanin-induced autophagy and a potential drug target for chemotherapeutic combination. These novel findings improve our understanding of gartanin-mediated autophagy as well as the mechanisms involved in its therapeutic role in hepatoma cancer patients.

\section{Acknowledgements}

The present study was supported (in part) by the Daegu University Research Grant, 2011.

\section{References}

1. Schweichel JU and Merker HJ: The morphology of various types of cell death in prenatal tissues. Teratology 7: 253-266, 1973.

2. Levine B and Yuan J: Autophagy in cell death: An innocent convict? J Clin Invest 115: 2679-2688, 2005.

3. Lieberman AP, Puertollano R, Raben N, Slaugenhaupt S, Walkley SU and Ballabio A: Autophagy in lysosomal storage disorders. Autophagy 8: 719-730, 2012.

4. Klionsky DJ, Abdalla FC, Abeliovich H, Abraham RT, AcevedoArozena A, Adeli K, Agholme L, Agnello M, Agostinis P, Aguirre-Ghiso JA, et al: Guidelines for the use and interpretation of assays for monitoring autophagy. Autophagy 8: 445-544, 2012.

5. He C and Klionsky DJ: Regulation mechanisms and signaling pathways of autophagy. Annu Rev Genet 43: 67-93, 2009.

6. Sinha S and Levine B: The autophagy effector Beclin 1: A novel BH3-only protein. Oncogene 27 (Suppl 1): S137-S148, 2008.

7. Funderburk SF, Wang QJ and Yue Z: The Beclin 1-VPS34 complex - at the crossroads of autophagy and beyond. Trends Cell Biol 20: 355-362, 2010.

8. El-Serag HB and Mason AC: Rising incidence of hepatocellular carcinoma in the United States. N Engl J Med 340: 745-750, 1999.

9. Parkin DM, Pisani P and Ferlay J: Global cancer statistics. CA Cancer J Clin 49: 31-64, 1999.

10. Josephs DH and Ross PJ: Sorafenib in hepatocellular carcinoma. Br J Hosp Med 71: 451-456, 2010.

11. Suksamrarn S, Komutiban O, Ratananukul P, Chimnoi N, Lartpornmatulee N and Suksamrarn A: Cytotoxic prenylated xanthones from the young fruit of Garcinia mangostana. Chem Pharm Bull 54: 301-305, 2006.

12. Jung HA, Su BN, Keller WJ, Mehta RG and Kinghorn AD: Antioxidant xanthones from the pericarp of Garcinia mangostana (Mangosteen). J Agric Food Chem 54: 2077-2082, 2006.
13. Kikuchi H, Ohtsuki T, Koyano T, Kowithayakorn T, Sakai T and Ishibashi M: Activity of mangosteen xanthones and teleocidin a-2 in death receptor expression enhancement and tumor necrosis factor related apoptosis-inducing ligand assays. J Nat Prod 73: 452-455, 2010.

14. Moon DO, Asami Y, Long H, Jang JH, Bae EY, Kim BY, Choi YH, Kang CH, Ahn JS and Kim GY: Verrucarin A sensitizes TRAIL-induced apoptosis via the upregulation of DR5 in an eIF $2 \alpha / \mathrm{CHOP}-d e p e n d e n t$ manner. Toxicol In Vitro 27: 257-263, 2013.

15. Colosetti P, Puissant A, Robert G, Luciano F, Jacquel A, Gounon P, Cassuto JP and Auberger P: Autophagy is an important event for megakaryocytic differentiation of the chronic myelogenous leukemia K562 cell line. Autophagy 5: 1092-1098, 2009.

16. Moon DO, Asami Y, Kim MO, Jang JH, Kim BY, Ahn JS, Kim GY and Yun SG: Xestospongin C induces monocytic differentiation of HL60 cells through activation of the ERK pathway. Food Chem Toxicol 55: 505-512, 2013.

17. Mizushima $\mathrm{N}$ and Yoshimori T: How to interpret LC3 immunoblotting. Autophagy 3: 542-545, 2007.

18. Li C, Capan E, Zhao Y, Zhao J, Stolz D, Watkins SC, Jin S and $\mathrm{Lu} B$ : Autophagy is induced in CD $4^{+} \mathrm{T}$ cells and important for the growth factor-withdrawal cell death. J Immunol 177: 5163-5168, 2006.

19. Jia G, Cheng G, Gangahar DM and Agrawal DK: Insulin-like grow th factor-1 and TNF-alpha regulate autophagy through c-jun $\mathrm{N}$-terminal kinase and Akt pathways in human atherosclerotic vascular smooth cells. Immunol Cell Biol 84: 448-454, 2006.

20. Ogata M, Hino S, Saito A, Morikawa K, Kondo S, Kanemoto S, Murakami T, Taniguchi M, Tanii I, Yoshinaga K, et al: Autophagy is activated for cell survival after endoplasmic reticulum stress. Mol Cell Biol 26: 9220-9231, 2006.

21. Chen JL, Lin HH, Kim KJ, Lin A, Forman HJ and Ann DK: Novel roles for protein kinase Cdelta-dependent signaling pathways in acute hypoxic stress-induced autophagy. J Biol Chem 283: 34432-34444, 2008.

22. Geeraert C, Ratier A, Pfisterer SG, Perdiz D, Cantaloube I, Rouault A, Pattingre S, Proikas-Cezanne T, Codogno P and Poüs C: Starvation-induced hyperacetylation of tubulin is required for the stimulation of autophagy by nutrient deprivation. J Biol Chem 285: 24184-24194, 2010.

23. Wei Y, Pattingre S, Sinha S, Bassik M and Levine B: JNK1mediated phosphorylation of $\mathrm{Bcl}-2$ regulates starvation-induced autophagy. Mol Cell 30: 678-688, 2008.

24. Wagner EF and Nebreda AR: Signal integration by JNK and p38 MAPK pathways in cancer development. Nat Rev Cancer 9: 537-549, 2009.

25. Puissant A, Robert G, Fenouille N, Luciano F, Cassuto JP, Raynaud S and Auberger P: Resveratrol promotes autophagic cell death in chronic myelogenous leukemia cells via JNK-mediated p62/SQSTM1 expression and AMPK activation. Cancer Res 70: 1042-1052, 2010.

26. Li DD, Wang LL, Deng R, Tang J, Shen Y, Guo JF, Wang Y, Xia LP, Feng GK, Liu QQ, et al: The pivotal role of c-Jun NH2-terminal kinase-mediated Beclin 1 expression during anticancer agents-induced autophagy in cancer cells. Oncogene 28: 886-898, 2009.

27. Surh YJ: Cancer chemoprevention with dietary phytochemicals. Nat Rev Cancer 3: 768-780, 2003.

28. Maiuri MC, Zalckvar E, Kimchi A and Kroemer G: Self-eating and self-killing: Crosstalk between autophagy and apoptosis. Nat Rev Mol Cell Biol 8: 741-752, 2007.

29. Shingu T, Fujiwara K, Bögler O, Akiyama Y, Moritake K, Shinojima N, Tamada Y, Yokoyama T and Kondo S: Stage-specific effect of inhibition of autophagy on chemotherapy-induced cytotoxicity. Autophagy 5: 537-539, 2009.

30. Wang QJ, Ding Y, Kohtz DS, Mizushima N, Cristea IM, Rout MP, Chait BT, Zhong Y, Heintz N and Yue Z: Induction of autophagy in axonal dystrophy and degeneration. J Neurosci 26: 8057-8068, 2006. 